\title{
CONSTRUCTIONS ARISING FROM NÉRON'S HIGH RANK CURVES
}

BY

M. FRIED ${ }^{1}$

\begin{abstract}
Many papers quote Néron's geometric construction of elliptic curves of rank 11 over $\mathbb{Q}[\mathbf{N}]-$ still, at the writing of this paper, the elliptic curves of highest demonstrated rank. The purported reason for the ordered display of "creeping rank" in $[\mathbf{P P}, \mathbf{G Z}, \mathbf{N a}$ and $\mathbf{B K}]$ is to make $[\mathbf{N}]$ explicit. Excluding $[\mathbf{B K}]$, however, these papers derive little from Néron's constructions. All show some lack of confidence in the details of $[\mathbf{N}]$.

The core of this paper $(\S 3)$, meets objections to $[\mathbf{N}]$ raised by correspondents. Our method adds a novelty as it magnifies the constructions of $[\mathbf{N}]$ - "generation of pencils of cubics from their singular fibers". This has two advantages: it displays (Remark 4.2) the free parameters whose specializations give high rank curves; and it demonstrates the existence of rank 11 curves through one appeal only to Hilbert's irreducibility theorem. That is, we have eliminated the unusual analogue of Hilbert's result that takes up most of $[\mathbf{N}]$. In particular $(\$ 4(\mathrm{c}))$, the explicit form of the irreducibility theorem in $[\mathbf{F r}]$ applies to give explicit rank 11 curves over $\mathbb{Q}$ : with Selmer's conjecture, rank 12.
\end{abstract}

1. Introduction. Throughout this paper $K$ is a field of characteristic zero, $\bar{K}$ a fixed algebraic closure of $K$. All algebraic sets are open subsets of projective sets (closed algebraic subsets of $\mathbb{P}^{n}$, projective $n$-space). For $W$, an algebraic subset of $\mathbb{P}^{n}$, a point of $W$ is an $n+1$-tuple with coordinates in $L$, a field containing $K$, that satisfy the polynomial equations describing $W$ : that is, a classical point (usually with coordinates in $\bar{K}$ ). The points of $W$ with coordinates in $K$ we denote by $W(K)$. We briefly review highlights from the subsections.

We have posted the dominant mathematical figure from $[\mathbf{N}]$-the auxiliary curve configuration-right at the top of $\S 2(\mathrm{a})$, which is a detailed description of the total space of singular cubics (in $\mathbb{P}^{2}$ ) suitably tangent to auxiliary curve configurations. Each irreducible singular cubic may be parametrized by a copy of $\mathbb{P}^{1}$. A weakened version of the parametrization may be extended over the whole family of singular cubics from \$2(a). Perhaps the most entertaining (and least elementary) point of $\S 2(b)$ is the necessity of weakening the parametrization property to obtain a global result.

To each member of one of the families of $\$ 2(b)$ we associate a base point cubic pencil $(\S 3(\mathrm{a}))$. Some member of one of these pencils has rank at least 11 (§4(c)following $[\mathbf{N}])$. Our elaborate construction removes gratuitous comments from $[\mathbf{N}]$

\footnotetext{
Received by the editors June 22, 1982.

1980 Mathematics Subject Classification. Primary 14H10, 14H25, 14K15; Secondary 14G25, 14H40.

'Supported by 1981 Summer N.S.F. Grant MCS 80-03253, and funds from Saarbrücken University in West Germany.
} 
about points in general position, and it demonstrates the nature of the many parameters which we are free to specialize to obtain a high rank curve.

Much of the uncertainty about $[\mathbf{N}]$ centers on the nature of the auxiliary curve deriving from a basepoint cubic pencil. In particular, we show its "generic" irreducibility in $\S 3(\mathrm{~b})$, thus meeting an objection to $[\mathbf{N}]$ of J. P. Serre voiced in private correspondence.

The remaining arguments implicit in $[\mathbf{N}]$ appear in $\$ 4$, with one difference. Our argument circumvents the need for Néron's version of Hilbert's irreducibility theorem for covers of an elliptic curve with infinitely many rational points. This enables us to apply the explicit form of Hilbert's theorem that appears in [Fr] to make the production of Néron's high rank curves, in great profusion, as explicit a process as one could ask.

Among others, A. Schinzel, J. P. Serre and H. Zimmer have discussed this project with, and to the benefit of, the author.

ADDED IN PROOF. The recent appearance of a rank 12 curve in [Me] compels us to remove our reliance on Selmer's conjecture to get families of rank 12 from Néron's method (last paragraphs of (3a) and of (4c)).

\section{The auxiliary curve configuration.}

(a) Singular cubics. Let $S$ be the subset of $\left(\mathbb{P}^{2}\right)^{4}$ consisting of 4-tuples $\left(P_{0}, P_{1}, P_{2}, P_{3}\right)$ such that no three of $\left\{P_{0}, P_{1}, P_{2}, P_{3}\right\}$ lie on a line. For a point $s \in S$ we obtain a diagram - the auxiliary curve configuration:

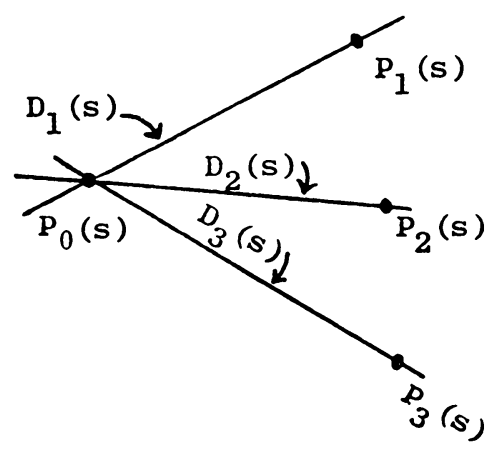

where $D_{i}(s)$ is the line joining $P_{0}(s)$ to $P_{i}(s), i=1,2,3$.

For each $s \in S$ consider $L_{s}$, the collection of cubics $\bigodot_{0}$ with the following properties:

(a) $\bigodot_{0}$ has at least one singular point;

(b) $\mathcal{C}_{0}$ is tangent to $D_{i}(s)$ at $P_{i}(s), i=1,2,3$; and

(c) the singular point of $\bigodot_{0}$ is distinct from $P_{i}(s), i=1,2,3$.

If $P_{4}$ is a point in $\mathbb{P}^{2}$, then the condition that a degree 3 homogeneous equation $f_{0}(x, y, z)=0$ defines a curve with a singular point at $P_{4}$ is really 3 linear conditions: $\left.f_{0}\right|_{P_{4}}=0, \partial f_{0} /\left.\partial x\right|_{P_{4}}=0$ and $\partial f_{0} /\left.\partial y\right|_{P_{4}}=0$ (the condition $\partial f_{0} /\left.\partial z\right|_{P_{4}}=0$ is automatic since $f_{0}$ is homogeneous). 
Consider a line $l$ through $P_{4}$ that is not entirely contained in the locus $f_{0}=0$. The intersection of $l$ and this locus consists of 3 points (counted with proper multiplicity). So there is but one, at most, further point $P(l)$ (of multiplicity one) in this intersection besides $P_{4}$. In particular, if the locus $f_{0}=0$ contains no lines (i.e., $f_{0}$ is irreducible over $\bar{K}), P_{4}$ is the only singular point.

For simplicity assume that $P_{4}=(x(4), y(4), z(4))$ with $y(4) \neq z(4)$. Then the following equations parametrize $l$ with the points $(u, v) \in \mathbb{P}^{1}$. Either:

$$
x=t \cdot u+x(4) \cdot v, \quad y=u+y(4) \cdot v, \quad z=u+z(4) \cdot v
$$

or

$$
x=u+x(4) \cdot v, \quad y=y(4) \cdot v, \quad z=z(4) \cdot v,
$$

where $t \in \bar{K}$. Change $u$ to $(1 / t) \cdot u$ in (a) and let $t=\infty$ to see that the lines through $P_{4}$ are parametrized by a copy of $\mathbb{P}^{1}, \mathbb{P}_{t}^{1}=\bar{K} \cup\{\infty\}$, equipped with the inhomogeneous uniformizing variable $t$. Thus, for any given value of $t$ (corresponding to a line $l$ ), we recover the residual point $P(l)$ by solving for $u / v(\neq 0)$ from the equation

$$
f_{0}(t \cdot u+x(4) \cdot v, u+y(4) \cdot v, u+z(4) \cdot v)=0 .
$$

LeMma 2.1. Suppose that $f_{0}(x, y, z)=0$ is an absolutely irreducible singular cubic curve defined over $K$. Then there are rational functions $x(t), \dot{y}(t), z(t) \in K(t)$, one, at least, of which is nonconstant and all of degree at most 3 , for which $f_{0}(x(t), y(t), z(t))$ $\equiv 0$. In particular, the function field of this curve is isomorphic to $K(t)$.

Proof. Under the hypotheses the curve $f_{0}(x, y, z)=0$ has exactly one singular point. Clearly, therefore, this singular point, $P_{4}$, must have coordinates in $K$. Thus, from (2.4), the residual intersection of the line of $(2.3)(a)$ with the curve has coordinates that we may express as rational functions in $K(t)$ of degree at most 3 . These are the rational functions sought in the statement of the lemma.

EXAMPLE 2.2. A family of singular cubics. A cubic equation in $x, y, z$ has $(0,0,1)$ as a singular point if and only if the $z^{3}, x \cdot z^{2}$ and $y \cdot z^{2}$ terms are missing. In particular,

$$
f_{a}(x, y, z)=(z+a \cdot x) \cdot y^{2}-x^{2} \cdot((1+2 \cdot a) \cdot x-a \cdot z)=0
$$

has $(0,0,1)$ as a singular point for each $a \in \bar{K}$. Since $f_{a}(t \cdot u, u, u+v)=0$,

$$
u / v=-\left(a \cdot t^{2}+1\right) /\left(-t^{3} \cdot(1+2 \cdot a)+a \cdot t^{2}+a \cdot t+1\right)=m(t) .
$$

We get a parametrization $(t \cdot m(t), m(t), m(t)+1)$, which, by multiplication by the denominator of $m(t)$, we can write as $\left(t \cdot\left(-a \cdot t^{2}-1\right),-a \cdot t^{2}-1,-t^{3} \cdot(1+2 \cdot a)\right.$ $+a \cdot t)$. The case $a=0$ is special: the singular point is cuspidal-rather than ordinary-and the parametrization $\left(t, 1, t^{3}\right)$ is exceptionally simple.

There are other exceptional values for $a$. For $a=-1, f_{a}(x, y, z)$ is reducible, but the singularity at $(0,0,1)$ is still ordinary. The specialization of $a$ to $\infty$ (by regarding $a$ as an inhomogeneous parameter for $\left.\mathbb{P}_{a}^{1}=\bar{K} \cup\{\infty\}\right)$ gives

$$
f_{\infty}(x, y, z)=x \cdot y^{2}-2 \cdot x^{3}-x^{2} \cdot z=x \cdot\left(y^{2}-2 \cdot x^{2}-x \cdot z\right) .
$$

Here, again, the corresponding curve has a cuspidal singular point at $(0,0,1)$. Excluding the points $a=0, \infty$ and -1 , the normalization of the curve $f_{a}(x, y, z)=0$ 
has exactly two points lying over the singular point of $f_{a}(x, y, z)=0$. The collection of these points forms a degree 2 cover of $\mathbb{P}_{a}^{1}-\{0,-1, \infty\}$ whose completion over $\mathbb{P}_{a}^{1}$ is ramified, exactly, over the points $a=0, \infty$.

Now we return to a description of $L_{s}$ for $s \in S$. For $\bigodot_{0} \in L_{s}$ let $P_{4}\left(\bigodot_{0}\right)$ be the singular point. Since $P_{4}\left(\bigodot_{0}\right) \notin\left\{P_{1}(s), P_{2}(s), P_{3}(s)\right\}$, the 3 tangency statements (6 linear conditions) and the singular point statement (3 linear conditions) add up to 9 linear conditions that determine the curve $C_{0}$. Thus, $L_{s}$ is a copy of $\mathbb{P}^{2}-$ $\left\{P_{1}(s), P_{2}(s), P_{3}(s)\right\}$ parametrizing a family of singular cubics indexed by their singular points. An extension of Example 2.2, however, allows us to make a further point about $L_{s}$ as a parameter space.

Use (2.3) to regard $t$ as a parameter for the lines through $(1,0,3)$ (i.e., take $\left.P_{4}=(1,0,3)\right)$. In parametric form, using the coordinates $(u, v) \in \mathbb{P}^{1}$, the line corresponding to $t \in \mathbb{P}^{1}$ is

$$
\begin{aligned}
& \text { (a) } x=t \cdot u+v, \quad y=u, \quad z=u+3 \cdot v, \quad \text { or } \\
& \text { (b) } x=u+v, \quad y=0, \quad z=3 \cdot v .
\end{aligned}
$$

Note. It is the line (b) that goes through $(0,0,1)$. For $f(x, y, z)=0$ representing a plane cubic $e$ not containing $(1,0,3)$, there is a natural degree 3 map $\bigodot \stackrel{\varphi}{\rightarrow} \mathbb{P}_{t}^{1}$ that maps $\mathfrak{p} \in \mathcal{C}$ to the value of $t$ corresponding to the line through $(1,0,3)$ and $\mathfrak{p}$. If $\mathcal{E}$ is nonsingular, the points of $\mathcal{C}$ that are ramified in this map exactly correspond to the points $\mathfrak{p}$ for which the line from $(1,0,3)$ to $\mathfrak{p}$ is tangent to $\mathcal{C}$. Let $e(\mathfrak{p} / \varphi(\mathfrak{p}))$ be the order of ramification of $\mathfrak{p}$. We obtain decisive information from the Riemann-Hurwitz formula [H, p. 301]

$$
2 \cdot(\operatorname{deg}(\varphi)+g(\mathcal{C})-1)=\sum_{\mathfrak{p}}(e(\mathfrak{p} / \varphi(\mathfrak{p}))-1),
$$

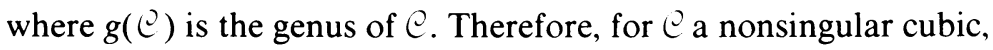

$$
\sum_{\mathfrak{p}}(e(\mathfrak{p} / \varphi(\mathfrak{p}))-1)=6,
$$

and for $\bigodot$ suitably "general" there are 6 lines tangent to $e$ from $(1,0,3)$.

If, on the other hand, $e$ is a singular cubic and $\mathcal{C}^{\prime}$ is its normalization, the natural map $\varrho^{\prime} \rightarrow \varrho$ induces $\varrho^{\prime} \stackrel{\varphi^{\prime}}{\rightarrow} \mathbb{P}_{t}^{\prime}$ with $\operatorname{deg}\left(\varphi^{\prime}\right)=3$ and $g\left(\varrho^{\prime}\right)=0$. Using (2.6) with $\mathfrak{p}^{\prime} \in e^{\prime}$,

$$
\sum_{\mathfrak{p}^{\prime}}\left(e\left(\mathfrak{p}^{\prime} / \varphi^{\prime}\left(\mathfrak{p}^{\prime}\right)\right)-1\right)=4 .
$$

Excluding the (at most 2) points of $\mathcal{C}^{\prime}$ that lie over the singular point of $\mathcal{C}$, the points of $e^{\prime}$ are in one-one correspondence with the points of $e$ with the singular point removed. Thus, for a "general" singular cubic there are 4 lines tangent to $\bigodot$ from $(1,0,3)$. Check that, if $a \neq 0$, the curve $f_{a}(x, y, z)=0$ (of Example 2.2) is in $L_{s(a)}$ for the following choice of coordinates for $s(a)$ : $P_{0}(s)=(1,0,3), P_{1}(s)=$ $(1,1,1), P_{2}(s)=(1,-1,1)$, and $P_{3}(s)$ whose $z$-coordinate is 1 and whose $x$-coordinate is $(-2 \cdot a) /\left(a^{2}-4 \cdot a-3\right)$. Indeed, the check is that the dot product of 
$\left.(\partial f / \partial x, \partial f / \partial y, \partial f / \partial z)\right|_{P_{i}(s)}$ with the vector $P_{i}(s)-P_{0}(s)$ is zero, $i=1,2,3$. Equivalently, if the $z$-coordinates are 1 , then the $x$-coordinates of the points of tangency from $(1,0,3)$ are given by

$$
\begin{aligned}
& m(x) \cdot(3 \cdot x-1) / 3+2 \cdot\left(x^{2} \cdot((1+2 \cdot a) \cdot x-a) \cdot(1+a \cdot x)\right)=0 \text { with } \\
& m(x)=a \cdot x^{2}((1+2 \cdot a) \cdot x-a)+x \cdot(-3 \cdot x \cdot(1+2 \cdot a)+2 \cdot a) \cdot(1+a \cdot x) .
\end{aligned}
$$

Note that the leading coefficient of this polynomial is 0 to see that it factors as

$$
x \cdot(x-1) \cdot\left(\left(\frac{1}{3} \cdot a^{2}-\frac{4}{3} \cdot a-1\right) \cdot x+\frac{2}{3} \cdot a\right) .
$$

Finally, observe that $(1,0,3)$ is on the curve $f_{a}(x, y, z)=0$ if and only if $a=1$. The remaining discussion is on the excluded case $a=0$ : the point $(0,0,1)$ is equal to $P_{3}(s)$ if and only if $a=0$.

Here is a geometric description of what happens as $a$ approaches 0 . Let $\varphi_{a}^{\prime}$ be the map called $\varphi^{\prime}$ earlier in the special case that $C_{\text {is }}$ the curve $f_{a}(x, y, z)=0$. Then, as $a$ approaches 0 , two of the branch points of the map $\varphi_{a}^{\prime}$ approach each other, and at $a=0$ the corresponding ramified points have coalesced to a single point $\mathfrak{p}_{0}^{\prime}$, with $e\left(\mathfrak{p}_{0}^{\prime} / \varphi_{0}^{\prime}\left(\mathfrak{p}_{0}^{\prime}\right)\right)=3$, on the normalization $\bigodot_{0}^{\prime}$ of $f_{0}(x, y, z)=0$; and $\mathfrak{p}_{0}^{\prime}$ is the only point of $e_{0}^{\prime}$ lying above $(0,0,1)$, the singular point of $f_{0}(x, y, z)=0$. Precisely because the cubic $f_{0}(x, y, z)=0$ violates condition $(2.2)(\mathrm{c})$ (and is therefore excluded from $L_{s(0)}$ ) and because this particular cubic figures in an important calculation in $\S 3(\mathrm{a})$, we wish to compactify $L_{s(0)}$ to $\bar{L}_{s(0)}$, a space with the following properties:

each point of $\bar{L}_{s(0)}$ corresponds to a cubic satisfying

$$
\text { properties }(2.2)(\mathrm{a}),(\mathrm{b}) \text {; }
$$

and

$$
\bar{L}_{s(0)} \text { also includes the cubic } f_{0}(x, y, z)=0 .
$$

One further computational observation makes it clear how to form $\bar{L}_{s(0)}$. The condition that the point $P_{1}(s(0))$ be a singular point on a cubic for which the line from $P_{0}(s(0))$ to $P_{1}(s(0))$ is tangent of order 3 to the cubic is 4 linear conditions on the coefficients of the cubic. The cubics which satisfy this condition, and also have the lines from $P_{0}(s(0))$ to $P_{2}(s(0))$ and to $P_{3}(s(0))$ as tangents, thus form a line in the space of cubics. It is clear, therefore, that $\bar{L}_{s(0)}$ should be identified with $\mathbb{P}^{2}$ with the points $P_{1}(s(0)), P_{2}(s(0))$ and $P_{3}(s(0))$ replaced by copies of $\mathbb{P}^{1}$. All the cubics corresponding to the points on the copy of $\mathbb{P}^{1}$ associated to $P_{1}(s(0))$ may, by hand, be seen to have the line $z=0$ (the line at infinity) as a tangent of multiplicity 3 . The extra linear condition stipulating the exact point of intersection with the line at infinity identifies the line at infinity with the copy of $\mathbb{P}^{1}$ associated to $P_{1}(s(0))$. In turn, the association of a point on the line at infinity with the line from $(0,0,1)$ to this point, identifies the copy of $\mathbb{P}^{1}$ with the directions at $P_{1}(s(0))$. Algebraic geometry therefore has a standard construction for $\bar{L}_{s}$ from $L_{s}: \bar{L}_{s}$ is the blow-up of $\mathbb{P}^{2}$ at the 3 points $P_{1}(s), P_{2}(s), P_{3}(s)[\mathbf{M}$, p. 309]. 
(b) A global parametrization. In this subsection we construct the most natural global parametrization of the family of singular cubics satisfying (2.2) (a) and (b) for some $s \in S$.

Continue the notation of $\S 2(a)$, including the spaces $\bar{L}_{s}$ for $s \in S$.

Definition 2.3. Let $f_{i}: X(i) \rightarrow Z$ be two functions with range $Z, i=1,2$. The fiber product $X(1) \times{ }_{Z} X(2)$ is the subset of $X(1) \times X(2)$ consisting of

$$
\left\{\left(x_{1}, x_{2}\right) \mid f_{1}\left(x_{1}\right)=f_{2}\left(x_{2}\right)\right\} \text {. }
$$

In the case that $X(2)$ is a subset of $Z$, the fiber product, denoted $\left.X(1)\right|_{X(2)}$ is called the fiber of $f_{1}$ over $X(2)$. If $X(1), X(2), Z$, and the graphs of $f_{1}$ and $f_{2}$ are quasiprojective sets (open subsets of projective sets), then so is $X(1) \times{ }_{Z} X(2)$ [M, p. 61 combined with the Segre morphism on p. 400], and so is the canonical projection map $\operatorname{pr}(i)$ : $X(1) \times{ }_{Z} X(2) \rightarrow X(i)$ onto the $i$ th coordinate, $i=1,2$.

First we construct a quasiprojective variety $\bar{L}$ with the following property: There is a morphism $\bar{L} \stackrel{\Lambda}{\rightarrow} S$ which naturally identifies the fiber over $s,\left.\bar{L}\right|_{s}$, with $\bar{L}_{s}$. On $S \times \mathbb{P}^{2}$ consider the subset $M=\left\{\left(s, P_{i}(s)\right) \mid i=1,2\right.$ or $\left.3, s \in S\right\}$. Then $\bar{L}$ is the blow-up of $S \times \mathbb{P}^{2}$ along $M$ [H, p. 163 and the proof of Theorem 7.17]. The map $\Lambda$ is the composite of the natural map from $\bar{L}$ to $S \times \mathbb{P}^{2}$ followed by projection onto the first factor. Since each point of $\bar{L}$ corresponds to a (singular) cubic in $\mathbb{P}^{2}$ there is a variety $e^{\text {sing }} \subset \bar{L} \times \mathbb{P}^{2}$ for which restriction of the projection to $\bar{L}$ gives $\operatorname{pr}(\bar{L})$ : $e^{\text {sing }} \rightarrow \bar{L}$ whose fiber $\left.e^{\text {sing }}\right|_{\wp}$ is the cubic that corresponds to the point $\wp \in \bar{L}$.

There is a maximal subset $\overline{L^{\text {ir }}}$ of $\bar{L}$ for which the cubics corresponding to points of $\bar{L}^{\mathrm{ir}}$ are irreducible. In fact, the computations of $\$ 2(a)$ can be embellished to give explicit equations for $\bar{L}-\overline{L^{\mathrm{i}}}$. But we do not do that here. Let $e^{\text {irsing }}$ be the fiber of $e^{\text {sing }}$ over $\overline{L^{1 r}}$ with the natural map

$$
\operatorname{pr}(\bar{L}): e^{\text {irsing }} \rightarrow \overline{L^{i r}} \text {. }
$$

This map has a section $\beta: \bar{L}^{\mathrm{ir}} \rightarrow e^{\mathrm{irsing}}$ that maps a point of $\bar{L}^{\mathrm{ir}}$ to the (unique) singular point in the fiber (i.e., $\operatorname{pr}(\bar{L}) \circ \beta=\mathrm{Id}$ ).

Each fiber $\left.e^{\text {irsing }}\right|_{\wp}$ has a canonical parametrization by a copy of $\mathbb{P}^{1}$ arising from the singular point on the fiber $(\$ 2(a))$. We understand this parametrization in a "global way" by considering a variety $\mathcal{R}$ with this property: There is a map $\Re \stackrel{\Delta}{\rightarrow} \mathbb{P}^{2}$ for which the fiber $\left.\Re\right|_{\mathbf{x}}, \mathbf{x} \in \mathbb{P}^{2}$, consists of a copy of $\mathbb{P}^{1}$ whose points are identified with the directions through $\mathbf{x}$ on $\mathbb{P}^{2}$. Form $\Re$ by taking $\mathbb{T}\left(\mathbb{P}^{2}\right)$, the tangent space to $\mathbb{P}^{2}$, remove the zero section, and projectivize what is left $([\mathbf{H}, \mathrm{pp} .162,429]$ - the result is called $\mathbb{P}\left(\mathbb{T}\left(\mathbb{P}^{2}\right)\right)$ ). Although each fiber of $\Delta$ is a copy of $\mathbb{P}^{1}, \Re$ itself is not isomorphic to $\mathbb{P}^{2} \times \mathbb{P}^{1}$ (e.g., if it were, the first Chern class of $\mathbb{T}\left(\mathbb{P}^{2}\right)$ would be 0 , but it is not $\left[\mathbf{H}\right.$, p. 433 combined with the computation of the canonical class on $\mathbb{P}^{2}$, p. 182]). The explicit parametrization of a singular cubic, by $\mathbb{P}_{t}^{1}$, given in (2.3) works only if the last two coordinates of the singular point are distinct. It is precisely because $R$ differs from $\mathbb{P}^{2} \times \mathbb{P}^{1}$ that there is no way to uniformly give such a parametrization (i.e., without a qualifying condition on the singular point).

Consider the map $\Sigma: \overline{L^{\mathrm{ir}}} \rightarrow \mathbb{P}^{2}$ that maps a point $\wp \in \bar{L}^{\text {ir }}$ to the singular point of the fiber of $\operatorname{pr}(\bar{L})$ over $\wp$. Form the fiber product $\bar{L}^{\mathrm{ir}} \times_{\mathbf{p}^{2}} \Re$ with respect to the maps 
$\Sigma$ and $\Delta$. The canonical parametrization of the fibers of $\operatorname{pr}(\bar{L})$ amount to a map

$$
\Psi: \overline{L^{\mathrm{r}}} \times_{\mathbf{p}^{2}} \Re \rightarrow e^{\text {ir sing }},
$$

where $\Psi$ is one-one over the open subset of $e^{\text {ir sing }}$ consisting of the complement of the image of the section $\beta$ of (2.8). This is the global parametrization of the title of this subsection.

Let $\left(\bigodot^{\text {irsing }} / \overline{L^{\text {ir }}}\right)^{n}$ be the fiber product of $e^{\text {irsing }} \rightarrow \bar{L}^{\text {ir }}$ taken $n$ times. Regard it as $n$-tuples of points on the fibers of $\operatorname{pr}(\bar{L})$. Thus, regard the fiber product of $\bar{L}^{\mathrm{ir}} \times_{\mathbf{p}^{2}}{ } \Omega$ over $\bar{L}$ taken $n$ times as a global parametrization of such $n$-tuples. Denote this last set, with its map to $\left(e^{\text {ir sing }} / \overline{L^{\text {ir }}}\right)^{n}$, by

$$
\Psi^{n}:\left(\overline{L^{\mathrm{ir}}} \times_{\mathbf{p}^{2}} \mathscr{R} / \overline{L^{\mathrm{ir}}}\right)^{n} \rightarrow\left(\bigodot^{\mathrm{ir} \text { sing }} / \overline{L^{\mathrm{r}}}\right)^{n} .
$$

\section{The general auxiliary curve.}

(a) Basepoint cubic pencils. Identify the space of cubics in $\mathbb{P}^{2}$ with the points of $\mathbb{P}^{9}$ by regarding the coordinates of a point in $\mathbb{P}^{9}$ as the coefficients, in some prespecified order, of the monomials in a cubic equation. Consider, especially, the locus $T$ of points that correspond to singular cubics. Since a cubic $f$ is singular if and only if one of the 6 (in general) points of intersection of $f=0$ and $\partial f / \partial x=0$ lies on $\partial f / \partial y=0$ - one local analytic condition-it is clear that $T$ is a subset of $\mathbb{P}^{9}$ of codimension 1: a hypersurface. Calculate its degree as the intersection multiplicity of $T$ with a line in $\mathbb{P}^{9}$.

A classical display of a specific elliptic pencil often uses the following notation:

$$
0=t \cdot f_{1}(x, y, z)+(1-t) \cdot f_{0}(x, y, z)=F(t, x, y, z) .
$$

Choose the homogeneous cubics $f_{0}$ and $f_{1}$ to be any two distinct members of the pencil so that $\mathbb{P}_{t}^{1}$ indexes members of the pencil (as in $\$ 2(a)$ ). It is typical to use singular members (i.e., $f_{0}=0$ or $f_{1}=0$, or both, are singular cubics) to generate the pencil. Since $f(t, x, y, z)=0, \partial f(t, x, y, z) / \partial x=0, \partial f(t, x, y, z) / \partial y=0$ (as a subspace of $(t, x, y, z)$-space) consists, in general, of 12 points, a general elliptic pencil has 12 singular (nodal) members.

EXAMPLE 3.1. A few special pencils:

(a) $A$ pencil lying entirely in $T$ : $0=t \cdot x^{3}+(1-t) \cdot z^{3}$ or $0=t \cdot z \cdot y^{2}+$ $(1-t) \cdot x^{2} \cdot(x-z)$. In the former case all members are, over $\bar{K}$, a product of lines. In the latter case most members are absolutely irreducible.

(b) A nearly trivial Weierstrass pencil: $0=t \cdot z \cdot y^{2}+(1-t) \cdot g_{1}(x, z)$, where $g_{1}(x, z)$ is a homogeneous cubic in $x$ and $z$ with no repeated roots. Over $\bar{K}$ all but a finite number of members are isomorphic to $z \cdot y^{2}+g_{1}(x, z)=0$, a cubic in Weierstrass form. The only singular members correspond to $t=0$ and $t=1$.

(c) A nontrivial Weierstrass pencil: $0=t \cdot\left(z \cdot y^{2}+g_{0}(x, z)\right)+(1-t) \cdot g_{1}(x, z)$, where $g_{0}(x, z)$ and $g_{1}(x, z)$ are nonzero homogeneous cubics with neither repeated nor common factors. The singular fibers are at $t=0$ and at the values of $t$ for which $G(t, x, z)=t \cdot g_{0}(x, z)+(1-t) \cdot g_{1}(x, z)$ has repeated factors (linear in $x$ and $\left.z\right)$. The equation $G(t, x, z)=0$ defines a curve $X$ in $\mathbb{P}_{t}^{1} \times \mathbb{P}^{1}$ (where the last factor has homogeneous coordinates $x$ and $z$ ). It is easy to check that $X$ is nonsingular. Thus, the values of $t$ for which $G(t, x, z)$ has repeated roots are precisely the branch points 
of the degree 3 natural projection $X \stackrel{p r_{1}}{\rightarrow} \mathbb{P}_{t}^{1}$. From $G(t, x, z)=0$, solve for $t$ as a rational function of $x / z$. That is, the function field of $X$ is generated by $x / z$, and $X$ is therefore of genus zero. An application of the Riemann-Hurwitz formula (2.6) shows that the number of branch points of the map $\mathrm{pr}_{1}$ does not exceed $2 \cdot(3-1)$ $=4$, and is exactly 4 for general $g_{0}$ and $g_{1}$.

Suppose that $0=t \cdot f_{1}+(1-t) \cdot f_{0}=F(t, x, y, z)$ is a cubic pencil for which $f_{0}=0$ and $f_{1}=0$ have 9 (distinct) points of intersection, denoted $Q(1), \ldots, Q(9)$. Then all members of the pencil have exactly these same 9 points of intersection with $f_{0}=0$. The following observation is well known. The reducible members of the pencil correspond exactly to partitions of $\{Q(1), \ldots, Q(9)\}$ into two subsets for which the points of the lst subset all lie on a conic and the points of the second subset all lie on a line.

Definition 3.2. Let $e_{0}$ be a singular (irreducible) cubic and let $(Q(1), \ldots, Q(8))=$ $\mathbf{Q}$ be 8 distinct points on $e_{0}$ (one of which may be the singular point of $e_{0}$ ). Denote by $\left|e_{0}, \mathbf{Q}\right|$ the linear system of cubics through the coordinates of $\mathbf{Q}$. In this form call the linear system a basepoint cubic pencil. From it, of course, we need to choose another member of the pencil to recover the classical description $F(t, x, y, z)=0$.

LEMMA 3.3. The general member of a basepoint cubic pencil is nonsingular.

Proof. Consider a basepoint cubic pencil $\left|\mathfrak{C}_{0}, \mathbf{Q}\right|$. From a theorem of Bertini [Z, p. 138], if each member of $\left|e_{0}, \mathbf{Q}\right|$ has a singular point, then this singular point must be a basepoint of the pencil. The coordinates of $\mathbf{Q}$ and one other point, $Q(9)$, constitute the basepoints of the pencil. If the lemma is false, then one of these is a singular point of $e_{0}$, and also of $\bigodot_{1}$, another member of the pencil. But this implies that the degree of the intersection $e_{0} \cap e_{1}$, as a divisor on $e_{1}$, exceeds 9 , contrary to Bezout's theorem.

Now consider the space $\left(e^{\text {irsing }} / \bar{L}^{\mathrm{ir}}\right)^{8}$ of $(2.10)$. Since this space consists of ordered 8-tuples of points on a family of singular cubics, denote by $\Delta^{(8)}$ the closed subset of 8-tuples with two or more equal coordinates. The complement of $\Delta^{(8)}$, together with the natural maps

$$
\left(e^{\text {ir sing }} / \overline{L^{\mathrm{ir}}}\right)^{8}-\Delta^{(8)} \stackrel{\operatorname{pr}(\bar{L})}{\rightarrow} \overrightarrow{L^{\mathrm{ir}}} \stackrel{\Lambda}{\rightarrow} S,
$$

therefore indexes distinct ordered 8-tuples of points on (irreducible) singular cubics together with an auxiliary curve configuration compatible with the cubic $((2.1)$ and (2.2)(a) and (b)). We call the diagram of (3.1) the space of basepoint cubic pencils (indexed by auxiliary curve configurations).

Finally, let $\mathfrak{p} \in\left(e^{\text {irsing }} / \overline{L^{\mathrm{ir}}}\right)^{8}-\Delta^{(8)}$ and let the picture of $(2.1)$ be the auxiliary curve configuration associated to $s=\Lambda \circ \operatorname{pr}(\bar{L})(\mathfrak{p})$. As in the remainder of $\S 2(\mathrm{a})$, $(2.2)(c)$ is not in force. We conclude this subsection with a description of three subsets of $\left(e^{\text {irsing }} / \overline{L^{1 \mathrm{r}}}\right)^{8}-\Delta^{(8)}$ that derive from $[\mathbf{N}]$. Let $Q_{i}^{\prime}(\mathfrak{p}), i=1,2,3$, be the intersection of $\bigcup_{0}(\mathfrak{p})=\operatorname{pr}(\bar{L})(\mathfrak{p})$ with $D_{i}(s)$ complementary to $P_{i}(s)$. Define: $\mathcal{K}(i)$, $i=1,2,3$, is the locus of those $\mathfrak{p} \in\left(e^{\text {irsing }} / \overrightarrow{L^{\text {ir }}}\right)^{8}-\Delta^{(8)}$ for which $Q(\mathfrak{p}, 1)=$ $Q_{1}^{\prime}(\mathfrak{p}), \ldots, Q(\mathfrak{p}, i)=Q_{i}^{\prime}(\mathfrak{p})$. So $\mathcal{N}(3) \subset \mathcal{X}(2) \subset \mathcal{N}(1)$. 
Néron's arguments for producing high rank elliptic curves over $\mathbb{Q}$ use the spaces $\Re(i)$. Indeed, both the work and the resulting ranks increase with $i$. Question: Is there an analogous space $\Re(4)$ ? Answer: Yes! We could have included a 4th line, $D_{4}(s)$, through $P_{0}(s)$ to which $\bigodot_{0}$ would also be tangent in the auxiliary curve configuration of (2.1). The resulting auxiliary curve $(\$ 3(\mathrm{~b}))$, however, corresponding to a point of $\Re(4)$, would be of genus 5 . Thus, it would not have an infinity of rational points with which to play Néron's game of applying a form of Hilbert's irreducibility theorem [N].

ADDED IN PROOF. Since, in $\S 4$, we circumvent this game, our method applies to T(4) to give rank 12 curves directly.

(b) Irreducibility and discussion of rank. We now construct the auxiliary curve $\mathscr{Q}(\mathfrak{p}, i)$ associated to a point $\mathfrak{p} \in \mathscr{N}(i)$ (note that if $\mathfrak{p} \in \mathcal{N}(3), \mathbb{Q}(\mathfrak{p}, 3)$ differs from $\mathbb{Q}(\mathfrak{p}, 2)$, even though $\mathfrak{p}$ is also in $\Re(2))$. Denote by $\left|\bigodot_{0}(\mathfrak{p}), \mathbf{Q}(\mathfrak{p})\right|$ the basepoint cubic pencil associated to $p$ (Definition 3.2). Again, let $\Lambda \circ \operatorname{pr}(\bar{L})(\mathfrak{p})=s$ (as in §3(a)) and let $D_{1}(s), D_{2}(s), D_{3}(s)$ be the lines through $P_{0}(s)$ in the auxiliary curve configuration of (2.1).

Here is the construction of $\mathbb{Q}(\mathfrak{p}, 2)$, a curve in $\left|\bigcup_{0}(\mathfrak{p}), \mathbf{Q}(\mathfrak{p})\right| \times D_{1}(s) \times D_{2}(s)$, for $\mathfrak{p} \in \mathcal{\varkappa}(2)$. For $m(i) \neq Q(\mathfrak{p}, i), i=1,2, m(i) \in D_{i}(s)$, there is a unique member $\mathfrak{C}_{m(i)} \in\left|e_{0}(\mathfrak{p}), \mathbf{Q}(\mathfrak{p})\right|$ for which $m(i) \in \mathfrak{e}_{m(i)} \cap D_{i}(s)$. Then $\mathbb{Q}(\mathfrak{p}, 2)$ is the closure of the set of triples

$$
\begin{aligned}
& \left\{\left(\bigodot^{\prime}, m(1), m(2)\right) \mid m(1) \in D_{1}(s), m(2) \in D_{2}(s),\right. \\
& \left.\quad m(i) \neq Q(\mathfrak{p}, i), i=1,2 \text {, and } \bigodot^{\prime}=\bigodot_{m(1)}=\bigodot_{m(2)}\right\} .
\end{aligned}
$$

By projection on the 1st factor we have a natural map

$$
\operatorname{pr}(1): \mathbb{Q}(\mathfrak{p}, 2) \rightarrow\left|\bigodot_{0}(\mathfrak{p}), \mathbf{Q}(\mathfrak{p})\right|
$$

and the fiber of this map over the member $\bigodot_{0}(p) \in\left|\bigodot_{0}(p), Q(p)\right|$ consists of a single point $\left(e_{0}(\mathfrak{p}), P_{1}(s), P_{2}(s)\right)$.

Another description of $\mathbb{Q}(\mathfrak{p}, 2)$ : Consider

$$
\varphi(\mathfrak{p}, i): D_{i}(s)-\{Q(\mathfrak{p}, i)\} \rightarrow\left|\bigcup_{0}(\mathfrak{p}), \mathbf{Q}(\mathfrak{p})\right|
$$

by $m(i) \rightarrow \bigodot_{m(i)}, i=1,2$. Then $\varphi(\mathfrak{p}, i)$ extends to a map $D_{i}(s) \rightarrow\left|\bigodot_{0}(\mathfrak{p}), \mathbf{Q}(\mathfrak{p})\right|$. Abbreviate $\left|C_{0}(\mathfrak{p}), \mathbf{Q}(\mathfrak{p})\right|$ to $|\mathfrak{p}|$. With respect to these maps, $\mathbb{Q}(\mathfrak{p}, 2)$ is the fiber product $D_{1}(s) \times_{|p|} D_{2}(s)$ (Definition 2.3).

EXAMPLE 3.4. Construction of $\mathscr{Q}(\mathfrak{p}, 2)$ for a special value of $\mathfrak{p} \in \Re(2)$. Let $C_{0}(\mathfrak{p})$ be the curve $z \cdot y^{2}-x^{3}=0$ (i.e., $a=0$ in Example 2.2). Then $\bigodot_{0}(\mathfrak{p})$ is in $\bar{L}_{s}$ for $s$ given (according to the material prior to $(2.7))$ by $P_{0}(s)=(1,0,3), P_{1}(s)=(1,1,1), P_{2}(s)$ $=(1,-1,1)$, and $P_{3}(s)=(0,0,1)$ (the singular point of $\left.e_{0}(p)\right)$. From the defining property of $\mathscr{X}(2)$ (end of $\S 3(\mathrm{a})$ ), compute that $Q(\mathfrak{p}, 1)=(-2,1,-8)$ and $Q(\mathfrak{p}, 2)=$ $(2,1,8)$ are (respectively) complementary to $P_{i}(s)$ on $D_{i}(3), i=1,2$. We "freely" let $Q(\mathfrak{p}, i)=\left(x(i), 1, x(i)^{3}\right), i=3, \ldots, 8$. 
Write the pencil $\left|\mathcal{C}_{0}(\mathfrak{p}), \mathbf{Q}(\mathfrak{p})\right|$ in the form

$$
\begin{aligned}
& (1-t) \cdot f_{0}+t \cdot f_{1}=0=(1-t) \cdot\left(z \cdot y^{2}-x^{3}\right) \\
& +t \cdot\left(a(2) \cdot x^{2} \cdot y+a(1) \cdot x \cdot y^{2}+a(0) \cdot y^{3}\right. \\
& \quad+z \cdot\left(b(2) \cdot x^{2}+b(1) \cdot x \cdot y+b(0) \cdot y^{2}\right) \\
& \left.\quad+z^{2} \cdot(c(1) \cdot x+c(0) \cdot y)+d(0) \cdot z^{3}\right) .
\end{aligned}
$$

Note that the cubic $f_{1}$ has no $x^{3}$ term. Therefore, each of the monomials of $f_{1}(x, y, z)$ contributes to unique terms of $f\left(x, 1, x^{3}\right)$. Conclude, if we take $d(0)=1$, that the coefficients of $f_{1}(x, y, z)$ are (up to sign) elementary symmetric functions in the $x$-coordinates of $Q(\mathfrak{p}, 1), \ldots, Q(\mathfrak{p}, 9)$, where $Q(\mathfrak{p}, 9)$ to the 9 th point of intersection of $f_{0}=0$ and $f_{1}=0$. Since the coefficient of $x^{8}$ in $f\left(x, 1, x^{3}\right)$ is 0 , the sum of these $x$-coordinates is 0 (i.e., $x(3)+x(4)+\cdots+x(9)=0$ ).

Denote by $u(1), \ldots, u(7)$ the elementary symmetric functions in $x(3), \ldots, x(9)$. Then the pencil $\left|\mathcal{C}_{0}(\mathfrak{p}), \mathbf{Q}(\mathfrak{p})\right|$ is of the form

$$
\begin{aligned}
(1- & t) \cdot\left(z \cdot y^{2}-x^{3}\right) \\
& +t \cdot\left(-(u(7)-4 \cdot u(5)) \cdot x^{2} \cdot y-4 \cdot u(6) \cdot x \cdot y^{2}+4 \cdot u(7) \cdot y^{3}\right. \\
& +z \cdot\left((u(4)-4 \cdot u(2)) \cdot x^{2}-(u(5)-4 \cdot u(3)) \cdot x \cdot y\right. \\
& \left.\left.+(u(6)-4 \cdot u(4)) \cdot y^{2}\right)+z^{2} \cdot((u(2)-4) \cdot x-u(3) \cdot y)+z^{3}\right)=0 .
\end{aligned}
$$

Let $i=1$ or 2 . Compute the $x$-coordinate of $m(t, i)$, denoted $x(9+i)$, for $t$ general and $m(t, i)$ a point of intersection of $D_{i}(s)-\{Q(\mathfrak{p}, i)\}$ and (3.3). Since the coordinates $(u+v, v, 3 \cdot u+v)$ parametrize $D_{1}(s)$, substitute $(x, 1,3 \cdot x-2)$ in (3.3) to conclude that $x(10)$ satisfies

$$
\begin{aligned}
(-(1-t)+ & (3 \cdot u(4)-3 \cdot u(2)-9) \cdot t) \cdot x(10)^{2} \\
+ & (2 \cdot(1-t)-((8 \cdot u(4)-2 \cdot u(2)-12) \\
& -(-u(7)+u(5)+3 \cdot u(3))) \cdot t) \cdot x(10) \\
+(-(1-t)+((-u(6)+4 \cdot u(4)-4) & -(-2 \cdot u(7)+2 \cdot u(3))) \cdot t)=0
\end{aligned}
$$

and, similarly, $x(11)$ satisfies

$$
\begin{aligned}
& (-(1-t)+(3 \cdot u(4)-3 \cdot u(2)-9) \cdot t) \cdot x(11)^{2} \\
& +(-2 \cdot(1-t)+((8 \cdot u(4)-2 \cdot u(2)-12) \\
& +(-u(7)+u(5)+3 \cdot u(3))) \cdot t) \cdot x(11) \\
& +(-(1-t)+((-u(6)+4 \cdot u(4)-4) \\
& +(-2 \cdot u(7)+2 \cdot u(3))) \cdot t)=0 \text {. }
\end{aligned}
$$

THEOREM 3.5. There is a nonempty (Zariski) open subset $U$ of $\Re(2)$ such that for $\mathfrak{p} \in U$ the auxiliary curve $\mathcal{Q}(\mathfrak{p}, 2)$ is absolutely irreducible.

Proof. Let $\mathfrak{p}^{\text {gen }}$ be a generic point of $\mathcal{T}(2)$. A general Bertini theorem argument (e.g., [FrS, Lemma 3.1]) shows that the theorem holds if $\mathscr{Q}\left(\mathfrak{p}^{\text {gen }}, 2\right)$ is absolutely 
irreducible, and this holds if $\mathscr{Q}(\mathfrak{p}, 2)$ is absolutely irreducible for some specific value of $\mathfrak{p}$. For the remainder of the proof take $\mathfrak{p}$ (and $\mathscr{Q}(\mathfrak{p}, 2)$ ) as in Example 3.4. In this case identify the pencil with $\mathbb{P}_{t}^{1}$. Use the discussion prior to Example 3.4 to identify $\mathbb{Q}(\mathfrak{p}, 2)$ with the fiber product of $\varphi(\mathfrak{p}, i): D_{i}(s) \rightarrow \mathbb{P}_{t}^{1}, i=1,2$.

The branch points of $\varphi(\mathfrak{p}, 1)$ (resp., $\varphi(\mathfrak{p}, 2))$ correspond to the values of $t$ obtained by equating to zero the discriminant of (3.4a) (resp., (3.4b)). One of the solutions is $t=0$. It is clear (for $x(3), \ldots, x(8)$ general) that the branch points of $\varphi(p, 1)$ and $\varphi(\mathfrak{p}, 2)$ different from $t=0$ are distinct. But this implies $\mathscr{Q}(\mathfrak{p}, 2) \rightarrow \mathbb{P}_{t}^{\prime}$ has 3 branch points, whereas the fiber product $D_{1}(s) \times_{|p|} D_{2}(s) \rightarrow \mathbb{P}_{t}^{1}$, if reducible, would have only two branch points. From this observation, conclude that $\mathscr{Q}(\mathfrak{p}, 2)$ is irreducible and, thus, the theorem holds.

For $\mathfrak{p} \in \mathscr{T}(3)$ define $\mathbb{Q}(\mathfrak{p}, 3)$ similarly, so that $\mathbb{Q}(\mathfrak{p}, 3)$ is the fiber product of 3 maps

$$
\varphi(\mathfrak{p}, i): D_{i}(s) \rightarrow\left|\bigodot_{0}(\mathfrak{p}), \mathbf{Q}(\mathfrak{p})\right|, \quad i=1,2,3 .
$$

THEOREM 3.6. In generalization to Theorem 3.5, there is a nonempty open subset $U$ of $\mathfrak{X}(3)$ such that for $\mathfrak{p} \in U$ the auxiliary curve $\mathfrak{Q}(\mathfrak{p}, 3)$ is absolutely irreducible.

Proof. As in the proof of Theorem 3.5, just find one $p \in \Re(3)$ for which the 3 maps of (3.5) have, excluding the common branch point corresponding to $\bigodot_{0}(p) \in$ $\left|\bigodot_{0}(\mathfrak{p}), \mathbf{Q}(\mathfrak{p})\right|$, distinct branch points. With one change only, take $\mathfrak{p}, \bigodot_{0}(\mathfrak{p})$ and $\mathbf{Q}(\mathfrak{p})$ as in Example 3.4: change $Q(\mathfrak{p}, 3)$, as dictated by $\mathfrak{p} \in \mathcal{N}(3)$, to $(0,0,1)=P_{3}(s)$ (the only point of intersection of $D_{3}(s)$ and $\bigodot_{0}(\mathfrak{p})$ ).

Follow the notation of Example 3.4. Denote by $u(1)^{\prime}, \ldots, u(5)^{\prime}$ the elementary symmetric functions in $x(4), \ldots, x(8)$. Since all members of the pencil $\left|\mathcal{C}_{0}(\mathfrak{p}), \mathbf{Q}(\mathfrak{p})\right|$ go through $(0,0,1)$, in the standard notation there is no $z^{3}$ term. Thus the pencil is of form

$$
\begin{aligned}
& (1-t) \cdot f_{0}+t \cdot f_{1}=0=(1-t) \cdot\left(z \cdot y^{2}-x^{3}\right) \\
& +t \cdot\left(a(2) \cdot x^{2} \cdot y+a(1) \cdot x \cdot y^{2}+a(0) \cdot y^{3}\right. \\
& +z \cdot\left(b(2) \cdot x^{2}+b(1) \cdot x \cdot y+b(0) \cdot y^{2}\right) \\
& \left.\quad+z^{2} \cdot(c(1) \cdot x+c(0) \cdot y)\right) .
\end{aligned}
$$

To determine the coefficients of the pencil apply the argument of Example 3.4 to recognize them (up to sign) as the elementary symmetric functions in $-2,2, x(4), \ldots, x(8)$, by taking the coefficient $c(1)$ to be 1 . Result: $\left|e_{0}(\mathfrak{p}), \mathbf{Q}(\mathfrak{p})\right|$ is

$$
\begin{aligned}
& (1-t) \cdot\left(z \cdot y^{2}-x^{3}\right) \\
& +t \cdot\left(-\left(u(5)^{\prime}-4 \cdot u(3)^{\prime}\right) \cdot x^{2} \cdot y-4 \cdot u(4)^{\prime} \cdot x \cdot y^{2}+4 \cdot u(5)^{\prime}\right. \\
& \cdot y^{3}+z \cdot\left(\left(u(2)^{\prime}-4\right) \cdot x^{2}-\left(u(3)^{\prime}-4 \cdot u(1)^{\prime}\right) \cdot x \cdot y\right. \\
& \left.+\left(u(4)^{\prime}-4 \cdot u(2)^{\prime}\right) \cdot y^{2}\right) \\
& \left.+z^{2} \cdot\left(x-u(1)^{\prime} \cdot y\right)\right)=0
\end{aligned}
$$


Since the coordinates $(u, 0,3 \cdot u+v)$ parametrize $D_{3}(s)$, the $x / z$-coordinate (distinct from 0 ) of the intersection of (3.6) and $D_{3}(s)$ satisfies

$$
-(1-t) \cdot(x / z)^{2}+t \cdot\left(\left(u(2)^{\prime}-4\right) \cdot(x / z)+1\right) .
$$

By computing the formulae corresponding to $(3.4 \mathrm{a}, \mathrm{b})$, conclude that the 3 maps of (3.5) have distinct branch points.

(c) Properties of general auxiliary curves. Several heuristic arguments of $[\mathbf{N}]$ (especially those applying forms of Hilbert's irreducibility theorem) give formal proofs if we use the spaces $\%(2)$ and $\mathcal{Y}(3)$ of $\S 3(b)$ as parameter spaces for the total spaces of elliptic pencils. The point of $\S 4(a)$ is to comment on the construction of such families. But first we list a few more properties of the "general" auxiliary curves $\mathcal{U}^{\prime}(\mathfrak{p}, 2)$ and $\mathcal{Q}(\mathfrak{p}, 3)$ for $\mathfrak{p} \in \mathfrak{N}(2)$ or $\mathfrak{\Re}(3)$. For $\mathfrak{p}$ a point of an algebraic variety defined over a field $K$, denote by $K(\mathfrak{p})$ the field generated over $K$ by inhomogeneous coordinates for $\mathfrak{p}$.

Proposition 3.7. For $p \in \mathcal{Y}(2)$ the curve $\mathscr{A}^{(}(\mathfrak{p}, 2)$ is singular at the point $\left(P_{1}(s), P_{2}(s)\right)$. If $\mathbb{A}^{\prime}(\mathfrak{p}, 2)^{\prime}$ is the normalization of $\mathbb{\leftrightarrow}^{\prime}(\mathfrak{p}, 2)$, then $\mathbb{A}^{\prime}(\mathfrak{p}, 2)^{\prime}$ has two distinct points lying over $\left(P_{1}(s), P_{2}(s)\right)$. Let $g(\hat{\Psi}(p, 2))$ be the geometric genus (the genus of a normalization) of $\mathcal{A}^{\prime}(\mathfrak{p}, 2)$. Then $g\left(\mathcal{A}^{\prime}(\mathfrak{p}, 2)\right)=0$ and $\mathcal{A}^{\prime}(\mathfrak{p}, 2)$ has infinitely many points in the field $\mathbb{Q}(\mathfrak{p})$.

In addition, there exists a nonempty (Zariski) open subset $U$ of $\mathscr{T}(3)$ such that for

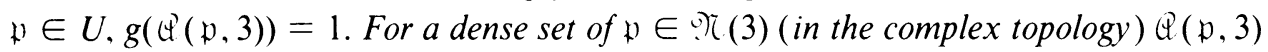
has infinitely many points in the field $\mathbb{Q}(\mathfrak{p})$.

Proof. Suppose that $X(i) \stackrel{\varphi(i)}{\rightarrow} \mathbb{P}_{t}^{1}, i=1,2$, are two finite morphisms of projective nonsingular curves. Let $\mathfrak{p}(i) \in X(i)$ have ramification index $e(i)$ over its image in $\mathbb{P}_{t}^{\prime}$. If $\min (e(1), e(2))>1$ and $\varphi(1)(p(1))=\varphi(2)(\mathfrak{p}(2))$, then the point $(\mathfrak{p}(1), \mathfrak{p}(2))$ is a singular point on the fiber product $X(1) \times_{\mathbb{p}_{t}^{\prime}} X(2)$ (Definition 2.3). Also, there are $(e(1), e(2))$ points on the normalization lying over $(p(1), p(2))$. Indeed, the result is local, and it follows from the case that $X(i)=\left\{(t, z(i)) \mid z(i)^{e(i)}=t\right\}$, an affine curve covering the affine $t$-line $\mathbf{A}_{t}^{1}, i=1,2$. Identify $X(1) \times_{\mathbf{A}_{t}^{1}} X(2)$ in this case with $\left\{(z(1), z(2)) \mid z(1)^{e(1)}=z(2)^{e(2)}\right\}$. Then the points on the normalization of $X(1)$ $\times_{\mathbf{A}_{t}, X(2)}$ above $(0,0)$ are in one-one correspondence with the irreducible factors over $\mathbb{C}$ of $z(1)^{e(1)}-z(2)^{e(2)}$. Apply this to $\mathbb{Q}(\mathfrak{p}, 2)=D_{1}(s) \times_{\mathbb{P}_{t}^{\prime}} D_{2}(s)$ and the point $\left(P_{1}(s), P_{2}(s)\right)$ to see that $\mathscr{Q}(\mathfrak{p}, 2)$ is singular and $\mathcal{G}(\mathfrak{p}, 2)^{\prime}$ has two points lying over $\left(P_{1}(s), P_{2}(s)\right)$.

To show that $g\left(\mathbb{Q}(\mathfrak{p}, 2)^{\prime}\right)=0$ it is sufficient to show this for $\mathfrak{p}$ general (the geometric genus is lower semicontinuous under specialization). From the remarks above, for $\mathfrak{p}$ sufficiently general, $Q(\mathfrak{p}, 2)^{\prime}$ is irreducible and a degree 4 cover of $\left|\mathfrak{e}_{0}(\mathfrak{p}), \mathbf{Q}(\mathfrak{p})\right|\left(\simeq \mathbb{P}_{t}^{1}\right)$ ramified over 3 points; and there are exactly two points of $\mathbb{Q}(\mathfrak{p}, 2)^{\prime}$ over each branch point of $\left|\mathfrak{e}_{0}(\mathfrak{p}), \mathbf{Q}(\mathfrak{p})\right|$. Apply (2.6):

$$
2 \cdot\left(4+g\left(\mathcal{Q}(\mathfrak{p}, 2)^{\prime}\right)-1\right)=3 \cdot 2=6,
$$

so $g\left(\mathfrak{Q}(\mathfrak{p}, 2)^{\prime}\right)=0$. Similarly, for $\mathfrak{p} \in \mathfrak{N}(3)$ suitably general, $\mathscr{Q}(\mathfrak{p}, 3)^{\prime}$ is irreducible (Theorem 3.6): a degree 8 cover of $\left|e_{0}(\mathfrak{p}), \mathbf{Q}(\mathfrak{p})\right|$ ramified over 4 points. And, over each of the branch points of $\left|\bigodot_{0}(\mathfrak{p}), \mathbf{Q}(\mathfrak{p})\right|$ there are exactly 4 points of $\mathscr{Q}(\mathfrak{p}, 2)^{\prime}$, 
each of ramification index 2 over the branch point. Thus, analogous to (3.7), $2 \cdot\left(8+g\left(\mathbb{Q}(\mathfrak{p}, 3)^{\prime}\right)-1\right)=4 \cdot 4$, so $g\left(\overleftrightarrow{Q}(\mathfrak{p}, 3)^{\prime}\right)=1$.

To conclude the proposition we have only to show that the curves $\mathbb{Q}(\mathfrak{k}, 2)$ and $\mathbb{P}(\mathfrak{p}, 3)$, clearly defined over $\mathbb{Q}(\mathfrak{p})$, have the declared rational point properties. The point $\left(P_{0}(s), P_{0}(s)\right)$ is a nonsingular point of $\mathscr{Q}(\mathfrak{p}, 2)$ (over the member of $\left|\bigodot_{0}(\mathfrak{p}), \mathbf{Q}(\mathfrak{p})\right|$ that passes through $\left.P_{0}(s)\right)$. Thus it determines a $\mathbb{Q}(\mathfrak{p})$-rational point of $\mathbb{Q}(p, 2)^{\prime}$. But, as is well known, a genus zero curve with one $\mathbb{Q}(\mathfrak{p})$-rational point is isomorphic (over $\mathbb{Q}(\mathfrak{p})$ ) to $\mathbb{P}^{1}$. Thus it, and $\mathbb{Q}(\mathfrak{p}, 2)$ also, both have infinitely many points in $\mathbb{Q}(\mathfrak{p})$.

Finally, consider $\mathfrak{p} \in \mathcal{X}(3)$ for which $\mathscr{Q}(\mathfrak{p}, 3)$ (and therefore $\left.\mathcal{Q}(\mathfrak{p}, 3)^{\prime}\right)$ is irreducible. Analogous to the preceding paragraph, $\left(P_{0}(s), P_{0}(s), P_{0}(s)\right)=\wp(0)$ defines a point of $\mathbb{Q}(\mathfrak{p}, 3)^{\prime}$ with coordinates in $\mathbb{Q}(\mathfrak{p})$. We divide the rest of the argument into parts.

Part 1. About the Picard components of a genus 1 curve. Let $e$ be a nonsingular curve of genus 1 and let $\varphi: \circlearrowright \rightarrow \mathbb{P}^{m}$ be a (finite) map of $\bigodot$ to $\mathbb{P}^{m}$ ( $m$ may be 1$)$ with $\varrho$ and $\varphi$ defined over a field $K$. Then $\varphi$ defines a linear system of divisors $|D|$ on $\varrho$ : the divisor $D$ is the formal sum of points of $C$ lying above the intersection of the image of $\mathcal{C}$ with a hypersurface in $\mathbb{P}^{m}$ not containing the image. Suppose that $|D|$ is a linear system of degree $d$ with no basepoints [H, p. 308].

Let Pic $(\mathcal{C})^{(n)}$ be the set consisting of linear equivalence classes of divisors on $C$ of degree $n$ : the $n$th connected component of the Picard group. The following facts appear in [L, Chapters II, VI]. Denote by $\bigodot^{(n)}$ the $n$th symmetric product of $C$ : the positive divisors of $e$ of degree $n$. The map that sends a positive divisor to its equivalence class

$$
\Psi^{(n)}: e^{(n)} \rightarrow \operatorname{Pic}(e)^{(n)}
$$

is called the Picard map of degree $n$. Since $\bigodot$ is defined over $K$, both Pic $(\bigodot)^{(n)}$ and $\Psi^{(n)}$ are projective and defined over $K$. For any divisor $D$ of degree $n$ let $[D]$ be the corresponding point of $\operatorname{Pic}(\bigodot)^{(n)}$. If $D$ is a $K$-rational divisor then $\left(\Psi^{(n)}\right)^{-1}([D])=|D|$ is isomorphic (over $K$ ) to $\mathbb{P}^{n-1}$. Even though $[D]$ is a $K$-rational point of $\operatorname{Pic}(C)^{(n)}$, it could be that $|D|$ contains no $K$-rational divisor. Note that $\mathrm{Pic}(\bigodot)^{(n)}$ is $K$-isomorphic to $\operatorname{Pic}(\Theta)^{(0)}$ by the map $T_{[D]}$ that sends $\left[D^{\prime}\right] \in \operatorname{Pic}(C)^{(n)}$ to $\left[D^{\prime}-D\right] \in \operatorname{Pic}(C)^{(0)}$. Of course, $\operatorname{Pic}(C)^{(0)}$ is an elliptic curve with a natural origin for its group structure, and it is isomorphic to $e$ (over $K$ ) if and only if $\mathcal{C}$ has a $K$-rational point.

Part 2. Picard interpretation of the infinite order of $\wp(0)$. For $\mathfrak{p} \in \mathcal{N}(3)$ suitably general, the hypotheses on $\mathcal{C}$ in Part 1 apply to $\mathscr{Q}(\mathfrak{p}, 3)^{\prime}$ over $K=\mathbb{Q}(\mathfrak{p})$, once we have explained how to get the degree 2 map $\mathscr{Q}(p, 3)^{\prime} \stackrel{\varphi}{\rightarrow} \mathbb{P}^{1}$. Since $p \in \mathscr{N}(3)$ it is also in $\mathscr{N}(2)$. From the fiber product interpretation of $\mathscr{Q}(\mathfrak{p}, 2)$ and $\mathscr{Q}(\mathfrak{p}, 3)$ (prior to Example 3.4), projection of $Q(p, 3)$ onto $\mathscr{Q}(\mathfrak{p}, 2)$ (the first two factors) induces a natural degree 2 map

$$
\mathbb{Q}(\mathfrak{p}, 3)^{\prime} \stackrel{\varphi^{\prime}}{\rightarrow} \mathbb{Q}(\mathfrak{p}, 2)^{\prime}
$$

From the discussion, however, prior to Part $1, \mathcal{Q}(\mathfrak{p}, 2)^{\prime} \simeq \mathbb{P}^{1}$. Take $\varphi^{\prime}=\varphi$. 
To show that $\mathbb{Q}(\mathfrak{p}, 3)^{\prime}$ has infinitely many $\mathbb{Q}(\mathfrak{p})$-rational points we need only show that $\operatorname{Pic}\left(\mathbb{Q}(\mathfrak{p}, 3)^{\prime}\right)^{(0)}$ has infinitely many $\mathbb{Q}(\mathfrak{p})$-rational points. We show, indeed, that for $[D]$ the equivalence class of degree 2 defined by $(3.9),[2 \cdot \wp(0)-D] \in$ $\operatorname{Pic}\left(\mathscr{Q}(\mathfrak{p}, 3)^{\prime}\right)^{(0)}$ has infinite order for $\mathfrak{p}$ suitably "general" in the complex topology.

Part 3. Variation of the point $[2 \cdot \wp(0)-D]$. The point $[2 \cdot \wp(0)-D]$ on $\operatorname{Pic}\left(Q(\mathfrak{p}, 3)^{\prime}\right)^{(0)}$ is a function of $\mathfrak{p}$. We show, for some values of $\mathfrak{p}$, that it is the origin of $\operatorname{Pic}\left(Q(p, 3)^{\prime}\right)^{(0)}$, and that for other values of $\mathfrak{p}$ it is not. Since the points of finite order on $\operatorname{Pic}\left(Q(p, 3)^{\prime}\right)^{(0)}$ are countable, this will show that the point $[2 \cdot \wp(0)-D]$ is of infinite order on $\operatorname{Pic}\left(\mathscr{Q}(\mathfrak{p}, 3)^{\prime}\right)^{(0)}$ for $\mathfrak{p}$ outside a subset of $\mathscr{T}(3)$ consisting of a countable union of subsets of codimension at least one.

Clearly, a degree 2 positive divisor of the form $2 \cdot q$ for some point $q \in \mathbb{Q}(\mathfrak{p}, 3)^{\prime}$ is linearly equivalent to $D$ if and only if $q$ is ramified in the map $\varphi^{\prime}$ of (3.9). Equally clearly, $\wp(0)$ is not ramified in general, but it is for some values of $\mathfrak{p}$ (e.g., for fixed $\bigcup_{0}(\mathfrak{p})$ and $s(\mathfrak{p})$ choose $\mathbf{Q}(\mathfrak{p})$ in (3.5) to achieve these properties). This concludes the proof of the theorem.

REMARK 3.8. The major point of the proof of Proposition 3.7 is the infinity of $\mathbb{Q}(\mathfrak{p})$-rational points on $\mathbb{Q}(\mathfrak{p}, 3)$. About the entire matter of $\S 3[\mathbf{N}, \mathrm{p} .487]$ says only (notation changed to ours): “Alors, si, les [coordinates of] $\mathbf{Q}(\mathfrak{p})$ ne satisfont pas à certaines conditiòns en nombre fini, le lieu $\mathbb{Q}(\mathfrak{p}, 3)$ est une courbe de genre 1 , qui possède un point rationnel $\wp(0)$ (associé à la courbe $\mathcal{C}_{0}(\mathfrak{p})$ de la pencil qui passe par $P_{0}(s)$ ). L' application du théorème d' irréductibilité (sous la forme de Hilbert) montre qu' on peut choisir les données de façon que $\wp(0)$ ne soit pas d' ordre fini dans le groupe des rationnels de $\mathbb{Q}(\mathfrak{p}, 3)$ et que les 11 points $\mathbf{Q}(\mathfrak{p})$ et $m(1), m(2), m(3)$ [as in §3(b)] soient indépendents dans le groupe des points de [the fiber of the pencil over a generic point of $\left.Q(p, 3)^{\prime}\right]$ ". We conclude this section with the proof of the italicized part of this last sentence. Note that we neither use Hilbert's irreducibility theorem, nor can we see how Néron intended to use it.

Suppose for $\mathfrak{p} \in \mathscr{T}(3)$ that $\mathscr{Q}(\mathfrak{p}, 3)$ satisfies the conclusion of Proposition 3.7. Each point $\mathbf{m}=(m(1), m(2), m(3)) \in \mathbb{Q}(\mathfrak{p}, 3)$ maps to a member $\bigodot_{\mathbf{m}}$ of $\left|e_{0}(\mathfrak{p}), \mathbf{Q}(\mathfrak{p})\right|$ and the coordinates of $\mathbf{Q}(\mathfrak{p})$ and $\mathbf{m}$ together give 11 points on $\bigodot_{\mathbf{m}}$, all defined over the field $\mathbb{Q}(\mathfrak{p}, \mathbf{m})$.

Proposition 3.9. Fix an irreducible cubic $\bigodot_{0}$ and $s \in S$ with $\bigodot_{0} \in \bar{L}_{s}$. Then, among those $\mathfrak{p} \in \mathcal{X}(3)$ for which $\bigcup_{0}(\mathfrak{p})=\bigodot_{0}$ and $s=\Lambda \circ \operatorname{pr}(\bar{L})(\mathfrak{p})$ (end of $\S 3(\mathrm{a})$ ) for $\mathfrak{p}$ suitably general (in the complex topology), $\mathbb{Q}(\mathfrak{p}, 3)$ contains a $\mathbb{Q}(\mathfrak{p})$-rational point $\mathbf{m}$ for which the member $\mathcal{C}_{\mathbf{m}}$ of $\left|\mathcal{C}_{0}(\mathfrak{p}), \mathbf{Q}(\mathfrak{p})\right|$ is an elliptic curve of rank over $\mathbb{Q}(\mathfrak{p})$ at least 11 .

Proof. Denote by $\left.\mathscr{N}(3)\right|_{\left(\Theta_{0}, s\right)}$ the collection of $\mathfrak{p} \in \mathcal{X}(3)$ for which $\bigodot_{0}(\mathfrak{p})=\bigodot_{0}$ and $s=\Lambda \circ \operatorname{pr}(\bar{L})(\mathfrak{p})$. Note that the proof of Proposition 3.7, Part 3, shows that for $\left.\mathfrak{p} \in \mathscr{N}(3)\right|_{\left(\Theta_{0}, s\right)}$ suitably general, $\mathbb{Q}(\mathfrak{p}, 3)$ has infinitely many $\mathbb{Q}(\mathfrak{p})$-rational points. Indeed, this holds for a generic point and let $\mathbf{m}\left(p^{\text {gen }}, 0\right)$ and $\mathbf{m}\left(\mathfrak{p}^{\text {gen }}, 1\right)$ be two points of $\mathbb{Q}(\mathfrak{p}, 3)^{\prime}$ for which $\mathbf{m}\left(\mathfrak{p}^{\text {gen }}, 1\right)$ is of infinite order in the group structure on $\mathbb{Q}(\mathfrak{p}, 3)^{\prime}$, using $\mathbf{m}\left(\mathfrak{p}^{\text {gen }}, 0\right)$ as a basepoint. Using the birational relation between $\mathcal{Q}(\mathfrak{p}, 3)$ and $Q(\mathfrak{p}, 3)^{\prime}$, regard $\mathbf{m}\left(\mathfrak{p}^{\text {gen }}, 1\right)$ as a 3 -tuple of points on $\bigodot_{\mathbf{m}\left(\mathfrak{p}^{\text {gen }}, 1\right)}$. 
Now, a similar argument to that of the proof of Proposition 3.7 suffices to show that $\bigodot_{\mathbf{m}\left(\mathfrak{p}^{\text {gen }}, 1\right)}$ is an elliptic curve of rank at least 11 over $\mathbb{Q}\left(\mathfrak{p}^{\text {gen }}\right)$.

We list two minor differences in the argument.

(i) Since $\bigodot_{m\left(\mathfrak{p}^{\mathrm{gen}}, 1\right)}$ is a cubic in $\mathbb{P}^{2}$ it is natural to take one of the flexes (a point at which a tangent to $\bigodot_{\mathbf{m}\left(\mathfrak{p}^{\mathrm{gen}}, 1\right)}$ meets this curve with multiplicity 3 , instead of the usual 2 - there are, counting multiplicity, 9 such flexes) as the basepoint for addition on $e_{\mathbf{m}\left(\mathfrak{p}^{\text {gen }}, 1\right)}$. This is just fine if one of these is $\mathbb{Q}\left(\mathfrak{p}^{\text {gen }}\right)$-rational. Otherwise, we go through the "Picard-type argument" taking $n=3$ in Part 1 of the proof of Proposition 3.7.

(ii) Similar to Part 3 of the proof of Proposition 3.7, show that by specialization of $\mathfrak{p}^{\text {gen }}$ to $\left.\mathfrak{p} \in \mathcal{R}(3)\right|_{\left(e^{0}, s\right)}$, the various coordinates of $\mathbf{m}(\mathfrak{p}, 1)$ and $\mathbf{Q}(\mathfrak{p})$ may be taken to be, or not to be (as is desired), flexes on $\bigodot_{m(p, 1)}$.

The rest is easy.

4. Ranks of specilized curves.

(a) Families of auxiliary curves. The spaces $\Re(2)$ and $\Re(3)$ of $\S 3(a)$ serve as parameter spaces for total families of cubic pencils. Lest the intricateness of this construction obstruct the main points, we supplant many details with a few explanations.

Consider an elliptic pencil

$$
0=t \cdot f_{1}(x, y, z)+(1-t) \cdot f_{0}(x, y, z)
$$

(or $\left|\mathcal{C}_{0}(\mathfrak{p}), \mathbf{Q}(\mathfrak{p})\right|$ with $\mathbf{Q}=(Q(1), \ldots, Q(8))$, whose coordinates are distinct points of $\bigodot_{0}$ ) corresponding to a point $\mathfrak{p} \in \mathcal{T}(2)$. The algebraic set $V$ given by (4.1) is a subset of $\mathbb{P}_{t}^{1} \times \mathbb{P}^{2}$. Since (4.1) determines $t$ as a function of $f_{1}(x, y, z) / f_{0}(x, y, z)$, except for those $(x, y, z)$ that are zeros of both $f_{0}$ and $f_{1}, V$ is birational to $\mathbb{P}^{2}$. Indeed [Sh, p. 160], if the ninth basepoint of the pencil, $Q(9)$, is distinct from the coordinates of $\mathbf{Q}$, then $V$ is isomorphic to $\mathscr{T}(\mathfrak{p}, 2), \mathbb{P}^{2}$ with $Q(1), \ldots, Q(9)$ blown up (§2(a)), equipped with a map $\mathcal{T}(\mathfrak{p}, 2) \stackrel{\alpha(\mathfrak{p})}{\rightarrow}\left|\bigodot_{0}(\mathfrak{p}), \mathbf{Q}(\mathfrak{p})\right|$ whose fiber over a member $t_{0} \in$ $\left|e_{0}(\mathfrak{p}), \mathbf{Q}(\mathfrak{p})\right|$ consists of the cubic of (4.1) corresponding to $t=t_{0}$.

If, however, $Q(9)=Q(i)$ for some $i, 1 \leqslant i \leqslant 8$, then the appropriate space $\mathcal{T}(\mathfrak{p}, 2)$ comes from the blow-up of a point on the exceptional fiber corresponding to $Q(i)$ on $\mathbb{P}^{2}$ with $Q(1), \ldots, Q(8)$ blown up.

We leave to the reader the use of these observations to construct algebraic varieties and algebraic maps defined over $\mathbb{Q}$ as follows:

$$
\mathcal{T}(2) \stackrel{\alpha}{\rightarrow} \mathcal{P}(2) \stackrel{\beta}{\rightarrow} \mathcal{K}(2)
$$

where the fiber $\left.\mathcal{P}(2)\right|_{\mathfrak{p}}$ of $\beta$ over $\mathfrak{p} \in \mathcal{T}(2)$ is identified with $\left|\bigodot_{0}(\mathfrak{p}), \mathbf{Q}(\mathfrak{p})\right|$, and the fiber of the diagram over $\mathfrak{p}$ is naturally identified with $\mathcal{\tau}(\mathfrak{p}, 2) \stackrel{\alpha(\mathfrak{p})}{\rightarrow}\left|\bigodot_{0}(\mathfrak{p}), \mathbf{Q}(\mathfrak{p})\right|$.

Similarly, using the fiber product definition of the auxiliary curve, it is easy to construct a finite morphism of algebraic varieties

$$
Q(2) \stackrel{\gamma}{\rightarrow} \mathscr{P}(2)
$$


where the fiber of the diagram over $p \in \mathcal{R}(2)$ is naturally identified with $\mathcal{H}^{\prime}(\mathfrak{p}, 2) \rightarrow$ $\left|\bigodot_{0}(\mathfrak{p}), \mathbf{Q}(\mathfrak{p})\right|$ (as in (3.2)). And, of course, there are analogous constructions with $\mathfrak{N}(3)$ replacing $\mathfrak{X}(2)$.

(b) Division point covers. Consider the fiber product $\mathcal{T}(3) \times_{\mathscr{Q P}(3)}(\mathcal{A}(3)$ arising from (4.2) and (4.3). Through projection on the second factor this fits in a diagram

$$
\widetilde{T}(3) \times_{\mathscr{P P}(2)}\left(\mathcal{P}^{\prime}(3) \rightarrow \mathcal{H}^{\prime}(3) \stackrel{\gamma}{\rightarrow} \mathscr{P}(3) \stackrel{\beta}{\rightarrow} \mathcal{T}(3)\right. \text {. }
$$

and for $\mathfrak{p} \in \mathcal{R}(3)$ the fiber of this diagram over $\mathfrak{p}$ is

$$
\widetilde{T}(p, 3) \times_{|p|} \mathbb{U}^{\prime}(p, 3) \rightarrow \mathcal{C}^{\prime}(p, 3) \rightarrow|p|,
$$

with $\left|e_{0}(p), \mathbf{Q}(\mathfrak{p})\right|$ denoted by $|p|$.

For $\mathcal{E}_{0}$ an irreducible cubic and $s \in S$ with $\mathcal{E}_{0} \in \bar{L}_{s}$, apply Proposition 3.9 to the generic point $\mathfrak{p}^{\text {gen }}$ of $\left.\mathscr{N}(3)\right|_{\left(\mathbb{E}_{0}, s\right)}=\left\{\mathfrak{p} \in \mathcal{R}(3) \mid \in_{0}(\mathfrak{p})=\bigodot_{0}\right.$ and $\left.s=\Lambda \circ \operatorname{pr}(\bar{L})(\mathfrak{p})\right\}$. Then (Proposition 3.9) $\mathbb{Q}\left(p^{\text {gen }}, 3\right)$ contains a $\mathbb{Q}\left(p^{\text {gen }}\right)$ point, $\mathbf{m}\left(p^{\text {gen }}\right)$, such that the fiber

$$
\mathcal{T}\left(p^{\text {gen }}, 3\right) \times_{\left|p^{\text {gen }}\right|}\left(\mathcal{Q}\left(p^{\text {gen }}, 3\right)=e_{\mathbf{m}\left(p^{\text {gen }}\right)}\right.
$$

has these properties: there is a $\mathbb{Q}\left(\mathfrak{p}^{\text {gen }}\right)$ point $\mathbb{Q}\left(\mathfrak{p}^{\text {gen }}, 0\right)$ on $\bigcup_{\mathbf{m}\left(\mathfrak{p}^{\mathrm{gen}}\right)}$ which gives a basepoint for a group structure on $\mathcal{E}_{\mathrm{m}\left(p^{\mathrm{gen}}\right)}$; and, in this group structure, the coordinates of $\mathbf{m}\left(\mathfrak{p}^{\text {gen }}\right)$ and $\mathbf{Q}\left(\mathfrak{p}^{\text {gen }}\right)$ generate a group, $\Gamma\left(\mathfrak{p}^{\text {gen }}\right)$, on $\bigodot_{\mathbf{m}\left(p^{\text {gen }}\right)}$ of rank at least 11 .

Following $[\mathbf{N}]$, let $\left\{\gamma\left(i, p^{\text {gen }}\right)\right\}_{i=1}^{t}$ be representatives of $\bigodot_{\mathbf{m}\left(\mathfrak{p}^{\mathrm{gen}}\right)}$ for the quotient $\Gamma\left(\mathfrak{p}^{\text {gen }}\right) / 2 \cdot \Gamma\left(\mathfrak{p}^{\text {gen }}\right)$ with $2 \cdot \Gamma\left(\mathfrak{p}^{\text {gen }}\right)=\left\{2 \cdot q \mid q \in \Gamma\left(\mathfrak{p}^{\text {gen }}\right)\right\}$. Symbolize the locus of points $q \in e_{\mathbf{m}\left(p^{g e n}\right)}$ that satisfy $2 \cdot \mathfrak{q}=\gamma\left(i, p^{\text {gen }}\right)$ by the symbol $\mathscr{D}_{\gamma\left(i, p^{g e n}\right)}$.

With specialization of $\mathfrak{p}^{\text {gen }}$ to $\left.\mathfrak{p} \in \mathcal{R}(3)\right|_{\left(\mathcal{E}_{0}, s\right)}$, the quantities $\mathbf{m}\left(\mathfrak{p}^{\text {gen }}\right)$, $\left\{\gamma\left(i, \mathfrak{p}^{\text {gen }}\right)\right\}_{i=1}^{t}$ specialize to $\mathbf{m}(\mathfrak{p}),\{\gamma(i, \mathfrak{p})\}_{i=1}^{t}$. Thus form an algebraic space $\mathbb{Q}_{\gamma(i)}$ and a finite morphism

$$
\left.\mathcal{Q P}_{\gamma(i)} \stackrel{\Delta(i)}{\rightarrow} \mathscr{X}(3)\right|_{\left(c_{0} . . s\right)}, \quad i=1, \ldots, t,
$$

such that the fiber $\left.\mathcal{O D}_{\gamma(i)}\right|_{\mathfrak{p}}$ over $\left.\mathfrak{p} \in \mathcal{N}(3)\right|_{\left(e_{0}, s\right)}$ is naturally identified with $\mathcal{O R}_{\gamma(i, \mathfrak{p})}$. The morphisms of (4.5) are the division poinc covers of $\left.\mathcal{T}(3)\right|_{\left(e_{0}, s\right)}$ (a similar construction works over $\mathfrak{R}(3))$ to which the title of this subsection refers. It is also clear that if $\mathcal{C}_{0}$ and $s$ are defined over $\mathbb{Q}$, as in $\S 4(c)$, then the algebraic sets and morphisms of (4.5) are also defined over $\mathbb{Q}$.

It is possible that $\mathscr{\mathscr { Q }}_{\gamma(i)}$ has more than one $\mathbb{Q}$-irreducible component, and it is also possible that some of these are of degree 1 over $\left.\mathscr{R}(3)\right|_{\left(\mathcal{C}_{0}, s\right)}$. Let $\left\{\mathscr{L}_{i}^{\prime}, i=1, \ldots, l\right\}$ be some listing of the $\mathbb{Q}$-irreducible components from the collection $\left\{\mathscr{Q}_{\gamma(i)}, i=1, \ldots, t\right\}$, for which, if $\mathscr{D}_{i}^{\prime} \subseteq \mathscr{D}_{\gamma(j)}$, the restriction $\left.\mathscr{Q}_{i}^{\prime} \stackrel{\Delta(i)^{\prime}}{\rightarrow} \mathscr{N}(3)\right|_{\left(e_{0}, s\right)}$ of $\Delta(j)$ to $\mathscr{Q}_{i}^{\prime}$ is of degree greater than 1 .

(c) Large ranks from an irreducibility result. Let $s \in S$ be a $\mathbb{Q}$-rational point and let $\mathcal{C}_{0} \in \bar{L}_{s}$ be an irreducible singular cubic defined over $\mathbb{Q}$. We show that a dense set of $\mathbb{Q}$-rational points of $\left.\mathscr{R}(3)\right|_{\left(e_{0}, s\right)}$ corresponds to elliptic curves over $\mathbb{Q}$ with rank at least 11. This is an application of Hilbert's irreducibility theorem as it 
appears in $[\mathbf{H i}]$ - no use is made of the unusual analogue for elliptic curves that takes up most of $[\mathbf{N}]$.

Consider $A\left(\bigodot_{0}\right)=\left\{\mathbb{Q}\right.$-rational points $\left.\mathfrak{p} \in \mathcal{K}(3)\right|_{\left(\varrho_{0}, s\right)} \mid$ the fiber of $\mathscr{Q}_{i}^{\prime}$ over $\mathfrak{p}$ has no $\mathbb{Q}$-rational points, $i=1, \ldots, l\}$.

TheOREM 4.1. For $\mathfrak{p} \in A\left(\bigodot_{0}\right)$ the elliptic curve $\bigodot_{\mathbf{m}(\mathfrak{p})}$ is defined over $\mathbb{Q}$ and has rank at least 11 . The points of $A\left(\mathcal{C}_{0}\right)$ are dense in $\left.\mathscr{R}(3)\right|_{\left(\mathcal{E}_{0}, s\right)}$. There is a similar result with or (3) $\left.\right|_{\left(\Theta_{0}, s\right)}$ replaced by $\Re(3)$.

Proof. A version of the statement about $\bigodot_{\mathbf{m}(\mathfrak{p})}$ for $\mathfrak{p} \in A\left(\bigodot_{0}\right)$ appears in $[\mathbf{N}, \mathrm{p}$. 486] without proof and in [N, 2, Chapter IV] with proof.

Since $\left.\Re(3)\right|_{\left(e_{0 . s)}\right.}$ is birational to $\mathbb{P}^{5}$, Hilbert's irreducibility theorem [Hi] implies that $A\left(\bigodot_{0}\right)$ is dense in $\left.\mathscr{N}(3)\right|_{\left(e_{0}, s\right)}$.

Remark 4.2. The space $S$ is birational to $\mathbb{P}^{8}$, the space $\bar{L}$ to $\mathbb{P}^{10}$ and the space $\Re(2)$ (resp., $\mathscr{N}(3)$ ) to $\mathbb{P}^{16}$ (resp., $\mathbb{P}^{15}$ ). Thus we can regard Néron's contruction as giving 16 (resp., 15) free variables that specialize to parametrize elliptic curves of rank 10 (resp., 11) over $\mathbb{Q}$.

Now apply the explicit form of Hilbert's irreducibility theorem in [Fr, §3] to explicitly find a dense subset $P$ of $A\left(\bigodot_{0}\right)$ deriving from arithmetic progressions of integers.

ADDED IN PROOF. Several correspondents have asked how this paper might give still higher rank curves. This is my best guess. Combine it with [BK] by trading off the free parameters that appear here for conditions that give higher rank.

\section{BIBLIOGRAPHY}

[BK] A. Brumer and K. Kramer, The rank of elliptic curves, Duke Math. J. 44 (1977), 716-743.

[Fr] M. Fried, On Hilbert's irreducibility theorem, J. Number Theory 6 (1974), 211-232.

[FrS] M. Fried and G. Sacerdote, Solving Diophantine problems over all residue class fields of a number field and all finite fields, Ann. of Math. (2) 104 (1976), 203-233.

[GZ] F. Grunewald and R. Zimmert, Über eineige rationale elliptische Kurven mit freiem Rang $\geqslant 8$,

J. Reine Angew. Math. 296 (1977), 100-107.

[H] R. Hartshorne, Algebraic geometry, Graduate Texts in Math., Springer-Verlag, New York, 1977.

[Hi] D. Hilbert, Über die Irreduzibilität ganzer rationaler Funktionen mit ganz zahligen Koeffizienten, J. Reine. Angew. Math. 110 (1892), 104-129.

[L] S. Lang, Abelian varieties, Interscience, New York, 1959.

[M] D. Mumford, Introduction to algebraic geometry, Harvard Univ. Notes, Cambridge, Mass., 1966.

[Na] K. Nakata, On some elliptic curves defined over $\mathbf{Q}$ of free rank $\geqslant 9$, Manuscripta Math. 29 (1979), No. 2-4, 183-194.

[N] A. Néron, Propriétés arithmétiques de certaines familles de courbes algébriques, Proc. Internat. Congress Math. (Amsterdam 1954), Vol. III, Noordhoff, Groningen; North-Holland, Amsterdam, 1956, pp. $481-488$.

$[\mathbf{N}, 2] \_$Problèmes arithmétiques et géométriques rattachès à la notion de rank $d$ 'une courbe algébrique dans un corps, Bull. Soc. Math. France 80 (1952), 101-166.

[PP] D. E. Penny and C. Pomerance, Three elliptic curves with rank at least seven, Math. Comp. 29 (1975), 965-967.

[Sh] I. R. Shafarevich, Lectures on minimal models and birational transformations of two dimensional schemes, Tata Inst. Fund. Res., Bombay, 1966.

[Z] O. Zariski, The theorem of Bertini on the variable singular points of a linear system of varieties, Trans Amer. Math. Soc. 56 (1944), 130-140.

[Me] J. F. Mestre, C. R. Acad. Sci. Paris 295 (1982), 643-644. (Added in proof.)

Department of Mathematics, University of California, Irvine, California 92717 